\title{
Development and Implementation of a Virtual Cell Culture Lab Practical for an Introductory BME Lab Course
}

\author{
Benjamin David (1), Faisal Masood (1), and Karin Jensen (1) \\ Department of Bioengineering, University of Illinois Urbana-Champaign, Urbana, IL, USA
}

(Received 30 June 2020; accepted 5 August 2020; published online 27 August 2020)

\begin{abstract}
The transition to remote learning in response to the COVID-19 pandemic necessitated the adaptation of an in-person cell culture lab practical to a virtual assessment in an introductory biomedical engineering lab course. The virtual lab practical was administered in the course LMS and implemented video, data analysis, and multiple-choice questions. Average student scores and grade distributions were comparable across in-person and virtual lab practical formats. Instructors observed fewer grading concerns for the virtual practical compared to previous in-person offerings. The virtual lab practical may be preferred over in-person lab practical in future offerings due to decreased student stress, lower cost, reduced required instructor time, and limited availability of equipment. Resources developed for the virtual practical, including video tutorials and a hemocytometer simulator to practice calculations, can be used by other educators and as supplements to existing course material.
\end{abstract}

Keywords-Laboratory, Cell culture, Online learning, Lab practical, Undergraduate.

\section{CHALLENGE STATEMENT}

In the field of biomedical engineering (BME), it is agreed that lab courses are essential to allow students to appreciate the connection between theoretical classes and the physical world. ${ }^{15}$ Previous work has also shown that an agentive BME lab class further improves learning outcomes, supporting hands-on labs as an impactful learning tool. ${ }^{13}$ At the University of Illinois Urbana-

Address correspondence to Karin Jensen, Department of Bioengineering, University of Illinois Urbana-Champaign, Urbana, IL, USA. Electronic mail: kjens@illinois.edu

Benjamin David and Faisal Masood contributed equally to the work.
Champaign, Cell and Tissue Engineering Lab is a required course for sophomores in bioengineering. The course provides the first exposure to wet lab work and mammalian cell culture in the curriculum. One of the learning outcomes for the course stated by the syllabus is "by the end of class, students will be able to culture bacterial and mammalian cells and use them in experiments." To assess student learning of mammalian cell culture technique, a lab practical is conducted at the end of the semester. In past semesters, students have performed basic cell culture techniques and calculations necessary to seed a new cell flask, and they were graded based on the proper implementation of the procedure and the use of aseptic technique. Prior to the COVID-19 outbreak, all instruction of cell culture was completed in the lab; however, the lab practical had yet to be conducted. In the process of moving to online instruction, the instructors of the Cell and Tissue Engineering Lab designed a virtual lab practical to assess the hands-on cell culture technique that was taught in the course. Although this assessment fails to address the students' physical technique, it requires students to think critically about technique. Here we describe the resources created for the online assessment, lessons learned, and provide these tools for broader use in the community.

\section{NOVEL INITIATIVE}

While practical skills (e.g., "the degree to which students can properly use scientific equipment, technology, and instrumentation, follow technical and professional protocols, and/or demonstrate proficiency in physical laboratory techniques, procedures, and 
measurements" ${ }^{\prime 3}$ ) are often critiqued as difficult or impossible to teach without traditional labs, ${ }^{7}$ most studies have shown equal or increased learning outcome achievement with virtual labs. ${ }^{3}$ Despite this, little work has explored using in-person lab training followed by virtual lab practical assessments. In response to moving the course to remote instruction necessitated by the COVID-19 pandemic in Spring 2020, we implemented a virtual lab practical to assess student cell culture proficiency after learning the techniques in an in-person instruction format. The approach to developing a virtual lab practical was chosen to reflect the content of the in-person practical.

In previous semesters, students were given a flask of 3T3-L1 mouse fibroblast cells and an abridged version of the course's cell culture protocol. Students were given $45 \mathrm{~min}$ to subculture the cells, manually count a sample using a hemocytometer, perform the calculations necessary to determine the viability and concentration of the cell solution, and seed the cell solution in a new flask at an instructor-specified density. Students completed the practical under the observation of an instructor but were not allowed to receive assistance.

For the virtual lab practical, we implemented a LMS-based (Canvas) assessment consisting of three phases: a video-based question set in which students were tasked with critiquing instructor cell culture technique, a data analysis question set requiring students to interpret microscope images and perform calculations, and a general multiple-choice question set (addressing topics of safety and purpose of reagents)
(Table 1). We recorded an array of short video clips depicting improper cell culture technique (Fig. 1), with examples based on common student errors observed by the instructors. Previous work has shown multimedia examination is most widely accepted by students if it mimics prior instruction. ${ }^{9}$ For this reason, we used this video question format to reflect the environment in which students would have taken the exam under normal circumstances, where instructors observed students use equipment and complete cell culture protocols. During the virtual lab practical, students were provided with the context of the video clip (what part of the protocol the video was depicting) and tasked with identifying what was done incorrectly and why the action was problematic. For example, in the sample video question provided, the students were informed that the experimenter is opening their biosafety cabinet at the beginning of cell culture. In the video, the experimenter opens the biosafety cabinet and introduces reagents and serological pipettes without first decontaminating the surface of the biosafety cabinet. In addition to identifying this technique error, the solution included that the error increases the risk of cell culture contamination (Online Resources 1, 2).

In developing the data analysis portion of the exam, we created a MATLAB script to randomly generate hypothetical images of cell-seeded hemocytometers (Fig. 2). The students were given these images and tasked with performing the same in-lab calculations necessary to seed new cell flasks. In addition, microscopy image analysis assessed student ability to esti-

TABLE 1. Virtual lab practical summary.

\begin{tabular}{lcccrr}
\hline Question format & Video & Hemocytometer count and calculation & Multiple choice & Evaluate cell images & Total \\
\hline Questions & 14 & 1 & 9 & 2 & 26 \\
Points & 140 & 50 & 90 & 20 & 300 \\
\hline
\end{tabular}

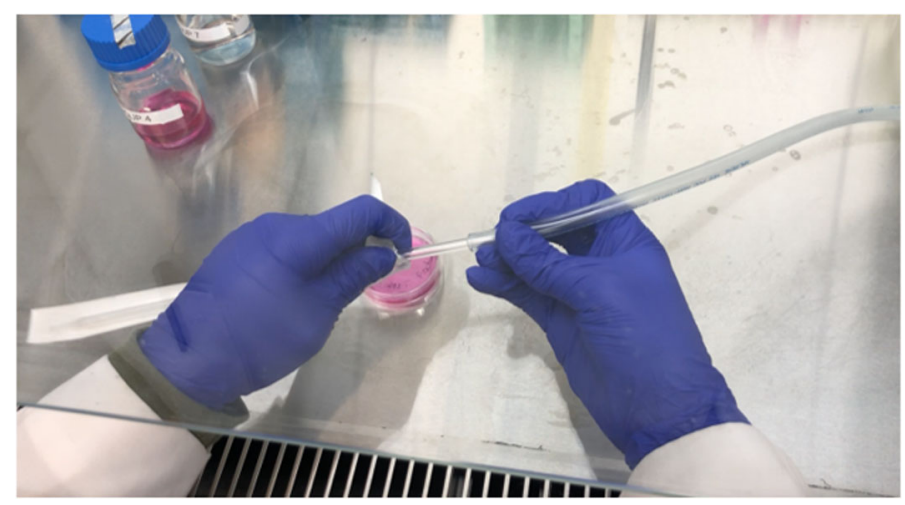

FIGURE 1. View of video clips showing cell culture procedure and technique. 
mate cell confluency and interpret results of a differentiation experiment performed in the lab prior to online learning (Fig. 3). Once more, we designed these data analysis exam questions to best resemble analytical tasks completed as part of the in-person lab practical. Lastly, multiple choice questions addressed lab safety and reagent function in the final portion of the exam.

It was essential that this online format simultaneously limited opportunities for academic dishonesty and maintained fairness amongst all students in the class. All questions were drawn from a larger question bank and randomized so that no two exams would be

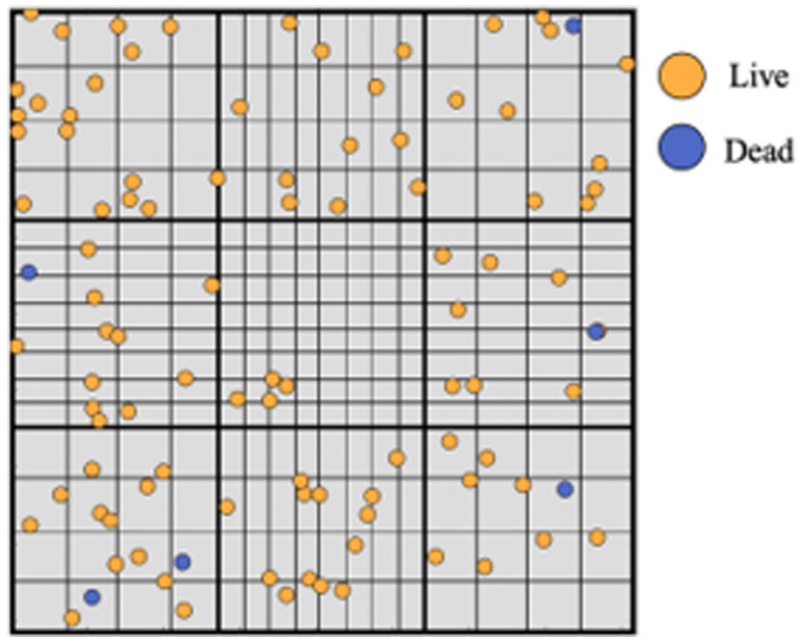

FIGURE 2. Hypothetical MATLAB hemocytometer images were used to assess student ability to count cells and perform related calculations. identical. Furthermore, the MATLAB script was used to generate many sets of hemocytometer images in order to limit the opportunity for sharing answers. Students were informed of the practical format (including all question formats) ahead of its administration. An example video question with the solution was created and shared with students before the lab practical (Online Resources 1) to help familiarize students with the video question format, which may be unfamiliar to students. The lab practical was made available to students for 1 week, and they were given 2-h to complete the assessment upon starting.

\section{REFLECTION}

Although it was impossible to test physical technique, the content of the virtual lab practical followed the concepts tested during the previously implemented in-person lab practical. Specifically, it required students to think critically about each step of the procedure and to interpret the same data they would have encountered in the lab. We observed that all students completed the virtual practical within the 2-h time and on average students completed the virtual practical in $1 \mathrm{~h}$ and $28 \mathrm{~min}$. To understand the potential impact of practical format on student grades, we compared virtual lab practical scores to in-person practical scores from previous semesters $[N=113,50$ (in-person), 63 (virtual)]. We assessed normality of the data by Shapiro-Wilk tests. ${ }^{17}$ The grades distributions for both virtual $(\mathrm{M}=92.09, \quad \mathrm{SD}=6.05)$ and in-person $(\mathrm{M}=91.86, \mathrm{SD}=10.16)$ practicals were non-normal

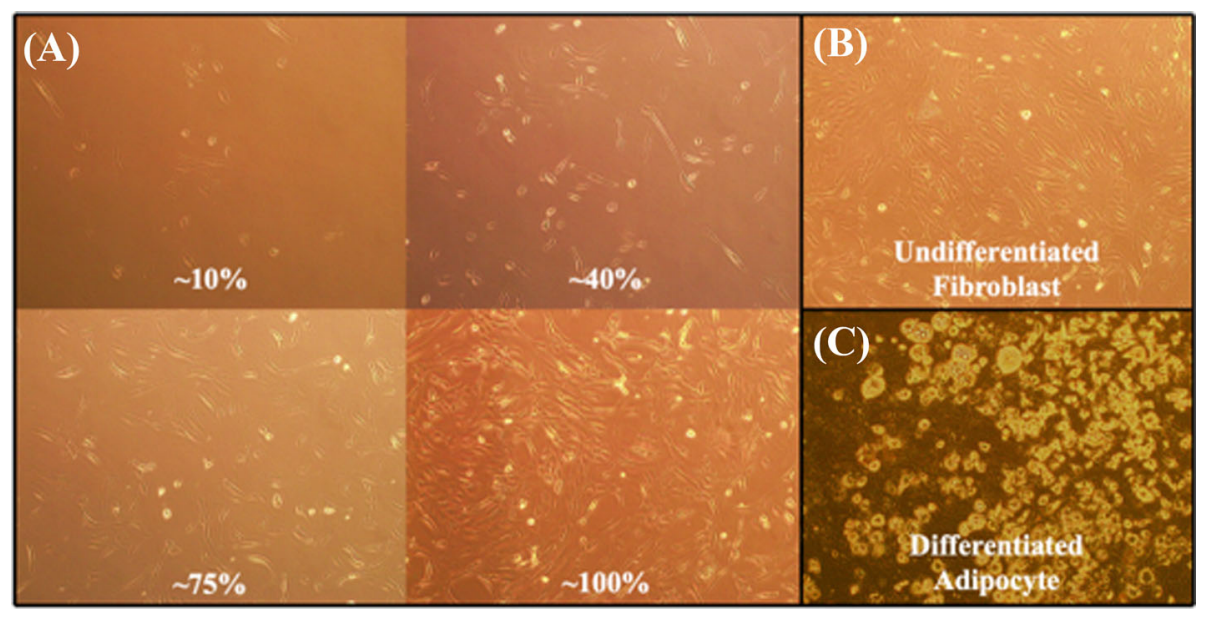

FIGURE 3. Example cell images used to assess (a) cell confluency estimation and (b, c) the success of a previously performed differentiation experiment. 


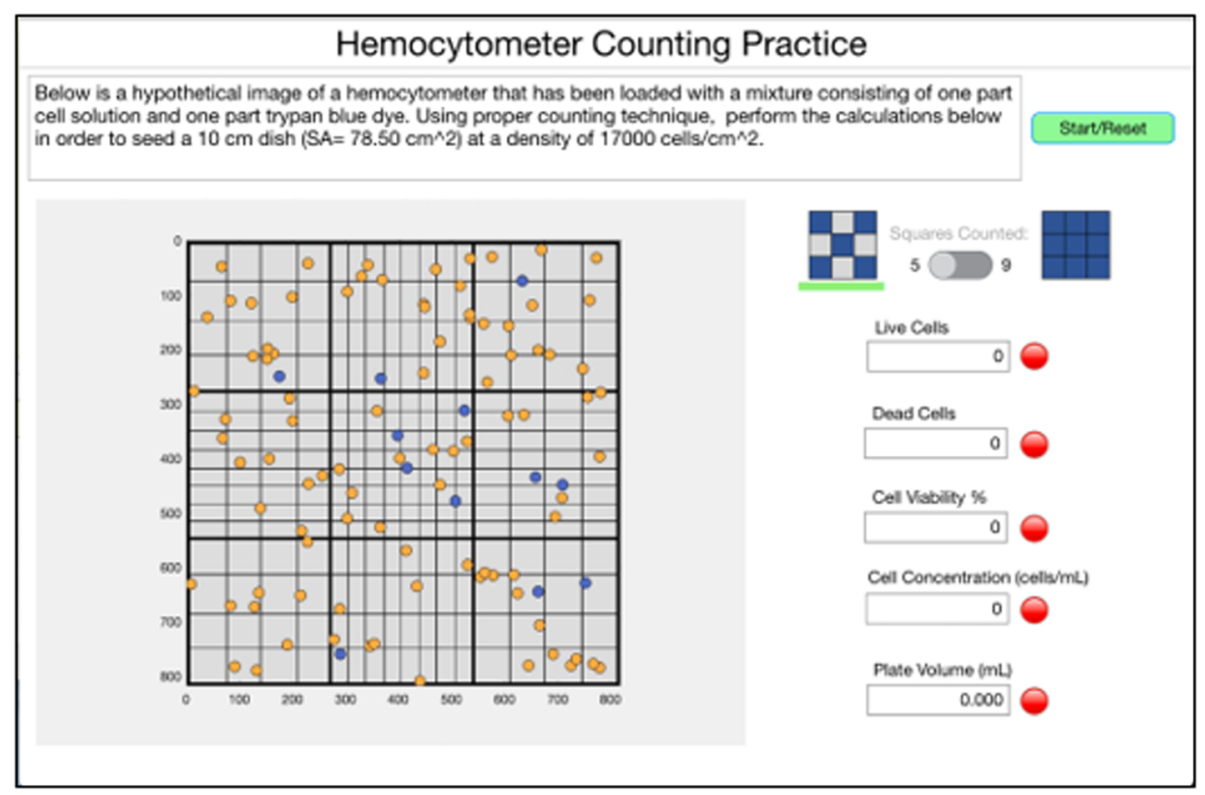

FIGURE 4. Hemocytometer counting practice application window. Pressing the Start/Reset button generates a new image as well as a new seeding flask and density. Lamps next to the input fields turn green when the correct answer has been entered.

$(p<0.001)$, indicating the data were suitable for nonparametric analysis. We tested average grades between groups using a Mann-Whitney $U$ test $^{12}$ and tested association of letter grade (with \pm ) distribution and practical format using a $\chi^{2}$ test of independence. ${ }^{11} \mathrm{We}$ found no significant difference in average scores between in-person and virtual practicals $(U=1358$, $z=-1.256, p=0.209$ ) and no significant association of grade distributions and format $\left(\chi^{2}(8\right.$, $\mathrm{N}=113)=13.086, \quad p=0.109), \quad$ suggesting that grades were comparable between in-person and virtual formats. After the exam was completed, we noted fewer re-grade requests and concerns about grading procedures compared to previous offerings of the inperson practical. This may indicate a strength of this format as compared to the traditional one, since in previous semesters students expressed concern toward the perceived subjectivity of live grading by multiple instructors across lab sections. We did observe that one video question yielded a sub- $25 \%$ success rate, and therefore the question was omitted accordingly to accommodate for this class-wide problem. This lowyield video question depicted a very subtle mistake that was poorly framed in the video clip, highlighting the need for question clarity when designing video questions for virtual assessments. Further, one challenge noted by a student was that given the randomization of the video questions, the questions on an individual exam were not necessarily sequential in following the protocol. This further emphasizes the importance of providing clear context for the video for questions in this format. Limitations of the current format include inability to test student handling of equipment (e.g., biosafety cabinet and microscope), but the format may provide more opportunity to test student understanding of the purposes of processes and technique (e.g., explaining why the technique was problematic) that were not directly assessed in previous offerings that assessed technique of performing the procedures.

Most available virtual cell culture lab resources are designed for teaching concepts and typically utilize either video narration ${ }^{14}$ or flash-based interactive programs. ${ }^{4}$ While others have shown that these platforms are effective in helping students develop a conceptual understanding, ${ }^{5}$ they do not address technique nor provide opportunities for troubleshooting offered by in-person lab instruction. While our approach does not fully replace the need for in-person instruction, the virtual lab practical requires students to be critical of observed technique in order to assess their understanding of the technique and protocols.

In recent semesters, we have been evaluating the effectiveness of the traditional lab practical in response to both teaching assistant and student feedback. Students have consistently commented that the traditional lab practical was a significant source of stress in previous semesters. Additionally, in-person labs have been critiqued as time and resource intensive, motivating the use of virtual or "blended" labs. ${ }^{19}$ Our course enrollment has grown considerably in recent years, with current enrollment at 50 students per semester. As a result, administering the traditional lab practical has become increasingly expensive both in terms of cost and instructor time, costing an estimated $\$ 16$ in re- 
agents per student and requiring 1 week of the semester, with each 1-h lab practical administered by an instructor for a single student at a time. Due to student stress, costs, and limitations in available biosafety cabinets and microscopes with a growing number of students, there may be reason to permanently administer the lab practical in the online format and to develop similar assessments for hybrid lab courses. ${ }^{18}$ While there is still a need for in-person instruction to effectively teach lab technique, virtual lab activities provided as supplements to in-person labs provide opportunities for repetition that support forming and understanding concepts, ${ }^{8}$ with studies showing improved course grades ${ }^{16}$ and conceptual understanding. ${ }^{1}$ Future work to assess the virtual lab practical format could also measure student self-efficacy as a result of an in-person vs. virtual lab practical. While we and others ${ }^{6}$ found that students preferred in-person compared to virtual labs (e.g., "I did not retain the information as well as if I was actually doing the lab."), future studies could assess student preferences, perceptions, and experiences of stress, comparing the virtual lab practical with the in-person format.

Prior to the onset of online learning, we recognized the benefit of providing supplemental online resources to students and had already produced multiple equipment tutorial videos (Online Resources 3, 4). These tutorial videos also included searchable transcription to increase accessibility. ${ }^{2,10} \mathrm{We}$ solicited student feedback and found that these resources helped them feel more comfortable in the lab. In future semesters, we plan to use the materials developed for this online lab practical as supplemental resources for in-person instruction. We have further developed the MATLAB script into a hemocytometer counting practice application, enabling future students to practice counting cells and performing calculations (Fig. 4) (Online Resource 5). We plan to continue developing creative online resources to improve the instruction and accessibility of the Cell and Tissue Engineering Lab. The resources associated with this initiative are freely provided for use at other institutions on our website and by contacting the instructor team.

\section{ELECTRONIC SUPPLEMENTARY MATERIAL}

The online version of this article (https://doi.org/10. 1007/s43683-020-00016-x) contains supplementary material, which is available to authorized users.

\section{ACKNOWLEDGMENTS}

The authors thank Holly Golecki for insightful feedback on the manuscript.

\section{CONFLICT OF INTEREST}

The authors declare no conflicts of interest.

\section{ETHICAL APPROVAL}

This work was approved by Blinded IRB \#14864.

\section{FUNDING}

This work was supported by the Department of Bioengineering at the University of Illinois UrbanaChampaign.

\section{CODE AVAILABILITY}

Available on https://github.com/bmdavid2/Hemoc ytometer_Practice.

\section{DATA AVAILABILITY}

All materials are available on our https://publish. illinois.edu/bioe202lab/ and upon request.

\section{REFERENCES}

\footnotetext{
${ }^{1}$ Abdulwahed M, Nagy ZK. Applying Kolb's experiential learning cycle for laboratory education. J Eng Educ. 2009;98:283-94.

${ }^{2}$ Angrave L, Jensen K, Zhang Z, Mahipal C, Mussulman D, Schmitz CD, Baird RT, Liu H, Wu MS, Kooper R. Improving student accessibility, equity, course performance, and lab skills: how introduction of ClassTranscribe is changing engineering education at the University of Illinois, presented at the ASEE annual conference \& exposition. 2020,

${ }^{3}$ Brinson JR. Learning outcome achievement in non-traditional (virtual and remote) versus traditional (hands-on) laboratories: a review of the empirical research. Comput Educ. 2015;87:218-37.

${ }^{4}$ Cell Culture Basics Virtual Lab. https://www.thermofishe r.com/us/en/home/global/forms/cell-culture-basics/cell-cul ture-basics-virtual-lab.html.

${ }^{5}$ Cherner Y, Cima M, Barone P, Van Dyke B, Lotring A. Interactive and adaptable cloud-based virtual equipment and laboratories for 21 st century science and engineering education. EPiC Ser Educ Sci. 2020;3:47-53.
} 
${ }^{6}$ Engum SA, Jeffries P, Fisher L. Intravenous catheter training system: computer-based education versus traditional learning methods. Am J Surg. 2003;186:67-74.

${ }^{7}$ Feisel LD, Rosa AJ. The role of the laboratory in undergraduate engineering education. J Eng Educ. 2005;94:12130 .

${ }^{8}$ Kirschner P, Meester M. The laboratory in higher science education: problems, premises and objectives. High Educ. 1988;17:81-98.

${ }^{9}$ Liu M, Papathanasiou E, Hao Y-W. Exploring the use of multimedia examination formats in undergraduate teaching: results from the fielding testing. Comput Hum Behav. 2001;17:225-48.

${ }^{10}$ Mahipal C, Angrave L, Xie Y, Chatterjee B, Wang H, Qian Z. "What did I just miss?!" Presenting ClassTranscribe, an automated live-captioning and Text-searchable lecture video system, and related pedagogical best practices, presented at the ASEE annual conference \& exposition, Tampa, FL.

${ }^{11} \mathrm{McHugh}$ ML. The Chi square test of independence. Biochemia Medica. 2013;23:143-9.

${ }^{12}$ Nachar N. The Mann-Whitney U: a test for assessing whether two independent samples come from the same distribution. Tutor Quant Methods Psychol. 2008;4:13-20.
${ }^{13}$ Newstetter WC, Behravesh E, Nersessian NJ, Fasse BB. Design principles for problem-driven learning laboratories in biomedical engineering education. Ann Biomed Eng. 2010;38:3257-67.

${ }^{14}$ Peña-Fernández A, Fenoy S, Evans MD, Sgamma T, Hurtado C, Izquierdo F, Randles M, Young C, Acosta L, Del Aguila C. Development of a virtual environment for teaching and learning biomedical techniques and equipment for the study of human pathogens. 2018 .

${ }^{15}$ Perreault EJ, Litt M, Saterbak A. Educational methods and best practices in BME laboratories. Ann Biomed Eng. 2006;34:209-16.

${ }^{16}$ Raineri D. Virtual laboratories enhance traditional undergraduate biology laboratories. Biochem Mol Biol Educ. 2001;29:160-2.

${ }^{17}$ Razali NM, Wah YB. Power comparisons of shapiro-wilk, kolmogorov-smirnov, lilliefors and anderson-darling tests. J Stat Model Anal. 2011;2:21-33.

${ }^{18}$ Son JY. Comparing physical, virtual, and hybrid flipped labs for general education biology. Online Learn. 2016;20:228-43.

${ }^{19}$ Toth EE, Morrow BL, Ludvico LR. Designing blended inquiry learning in a laboratory context: a study of incorporating hands-on and virtual laboratories. Innov Higher Educ. 2009;33:333-44. 